\title{
1 Machine Learning Models Identify Inhibitors of SARS-CoV-2
}

3 Victor O. Gawriljuk ${ }^{1}$, Phyo Phyo Kyaw Zin ${ }^{2}$, Daniel H. Foil ${ }^{2}$, Jean Bernatchez ${ }^{3}$, Sungjun

4 Beck $^{3}$, Nathan Beutler ${ }^{4}$, James Ricketts ${ }^{4}$, Linlin Yang ${ }^{4}$, Thomas Rogers ${ }^{4,5}$, Ana C. Puhl ${ }^{2}$,

5 Kimberley M. Zorn², Thomas R. Lane ${ }^{2}$, Andre S. Godoy ${ }^{1}$, Glaucius Oliva ${ }^{1}$, Jair L.

6 Siqueira-Neto $^{3}$, Peter B. Madrid ${ }^{6}$ and Sean Ekins ${ }^{2^{*}}$

8 'São Carlos Institute of Physics, University of São Paulo, Av. João Dagnone, 1100 -

9 Santa Angelina, São Carlos - SP, 13563-120, Brazil

$11{ }^{2}$ Collaborations Pharmaceuticals, Inc., 840 Main Campus Drive, Lab 3510, Raleigh, NC 12 27606, USA.

$14{ }^{3}$ Skaggs School of Pharmacy and Pharmaceutical Sciences, University of California 15 San Diego, La Jolla, California, 92093, USA.

$17 \quad{ }^{4}$ The Scripps Research Institute, La Jolla, California, 92093, USA.

$19{ }^{5}$ School of Medicine, University of California San Diego, La Jolla, California, 92093, USA.

$22{ }^{6}$ SRI International, 333 Ravenswood Avenue, Menlo Park, CA 94025, USA. 
bioRxiv preprint doi: https://doi.org/10.1101/2020.06.16.154765; this version posted June 16, 2020. The copyright holder for this preprint (which was not certified by peer review) is the author/funder, who has granted bioRxiv a license to display the preprint in perpetuity. It is made available under aCC-BY-NC-ND 4.0 International license.

23 *Corresponding Author sean@collaborationspharma.com

24 Key words: SARS-CoV-2, COVID-19, Machine learning, drug discovery, Bayesian 
31 have been selected for in vitro testing by several groups already. These have led to a

32 growing database of molecules with in vitro activity against the virus. Machine learning

33 models can assist drug discovery through prediction of the best compounds based on

34 previously published data. Herein we have implemented several machine learning

35 methods to develop predictive models from recent SARS-CoV-2 in vitro inhibition data

36 and used them to prioritize additional FDA approved compounds for in vitro testing

37 selected from our in-house compound library. From the compounds predicted with a

38 Bayesian machine learning model, CPI1062 and CPI1155 showed antiviral activity in

39 HeLa-ACE2 cell-based assays and represent potential repurposing opportunities for

40 COVID-19. This approach can be greatly expanded to exhaustively virtually screen

41 available molecules with predicted activity against this virus as well as a prioritization

42 tool for SARS-CoV-2 antiviral drug discovery programs. The very latest model for

43 SARS-CoV-2 is available at www.assaycentral.org. 


\section{Introduction}

In December 2019, several cases of pneumonia with unknown etiology started to

50 arise in Wuhan, China. A new betacoronavirus was identified and named SARS-CoV-2

51 due to high similarity with previous SARS-CoV ${ }^{1,2}$. This virus causes the disease which

52 has been called COVID-19 ${ }^{3}$.Since then, SARS-CoV-2 has rapidly spread worldwide

53 prompting the World Health Organization to declare the outbreak a pandemic, with more

54 than 1.5 million cases confirmed in less than 100 days. ${ }^{4}$ The high infection rate has also

55 caused considerable stress on global healthcare systems leading to more than 400,000

56 deaths from COVID-19.

The SARS-CoV-2 pandemic started a worldwide effort to discover a treatment

58 that could prevent further COVID-19 deaths and decrease the number and length of

59 hospitalization ${ }^{5}$. Drug repurposing is one of the main strategies being used to accelerate

60 this as most preclinical stages are removed and a promising drug could move directly

61 into phase II clinical studies or beyond by using an approved, safe drug ${ }^{6,7}$. So far, most

62 SARS-CoV-2 inhibition studies rely on small to medium scale assays with high

63 throughput screens (HTS) campaigns testing specific FDA-approved drugs and

64 compounds that have previously shown inhibition against different betacoronaviruses or

65 specific antiviral targets ${ }^{8-16}$.

66 Quantitative Structure Activity Relationship (QSAR) analyses from previous in

67 vitro data has been widely used to assist drug discovery in both industry and

68 academia $^{17}$. In the past few years the rise of machine learning has also expanded to

69 drug discovery, with different methods being implemented in a wide range of areas from

70 predicting synthetic routes to biological activity ${ }^{18,19}$. Many examples show that 
71 prioritizing compounds from machine learning and QSAR models can increase the

72 success rate and save resources ${ }^{17}$. Here we have implemented several machine

73 learning methods to develop predictive models from recent SARS-CoV-2 in vitro

74 inhibition data and used them to prioritize compounds for in vitro testing of different

75 compound libraries. These efforts will add to the list of $>200$ drugs and vaccines under

76 assessment elsewhere and which is continually growing ${ }^{20}$.

78 Materials and Methods

\section{Data Curation}

81 Data from recent drug repurposing campaigns for SARS-CoV-2 were used to

82 build a dataset from whole cell inhibition assays ${ }^{8,9,12,14,15}$. In assays with several

83 Multiplicity of Infection (MOI) the one closer to the whole dataset was chosen. In

84 machine learning model generation, duplicate compounds with finite activities are

85 averaged into a single entry. Due to the potential for diminished activity, when duplicate

86 compounds were present, only the most active one was retained in the dataset.

87 Additionally, compounds with ambiguous dose-response curves were discarded.

88 Datasets were built with Molecular Notebook (Molecular Materials Informatics, Inc). In

89 order to evaluate the model performance on an external testing set, a total of 30

90 molecules was collated from different studies ${ }^{11,21-25}$.

91

\section{Assay Central $^{\mathrm{TM}}$}


The Assay Central ${ }^{\mathrm{TM}}$ software $(A C)$ has been previously described ${ }^{19,26-34}$. AC

94 employs a series of rules for the detection of problem data for automated structure

95 standardization to generate high-quality data sets and Bayesian machine learning

96 models capable of predicting potential bioactivity for proposed compounds. AC was

97 used to prepare and merge data sets, as well as generate Bayesian models using the

98 ECFP6 descriptor and five-fold cross validation. During model generation, training

99 compounds are standardized (i.e. salts were removed, corresponding acids

100 neutralized), and thresholds for binary activity classification are applied to optimize

101 internal five-fold cross validation metrics. For predictions, AC workflows assign a

102 probability score and applicability score to prospective compounds according to a user-

103 specified model, with prediction scores greater than 0.5 considered active.

104

105 Additional Machine Learning Methods

106

Additional Machine learning algorithms including Bernoulli Naïve Bayes (bnb),

107 AdaBoost Decision trees (ada), Random Forest (rf), support vector classification (svc),

108 k-Nearest Neighbors (knn) and Deep Learning (DL) were also implemented with ECFP6

109 fingerprints and five-fold cross validation. Details for the development of these models

110 was previously described in detail in our earlier articles ${ }^{28,32,35}$. Bayesian models were

111 also generated with Discovery Studio (Biovia, San Diego CA) using ECFP6 descriptors

112 where the top and bottom scoring fingerprints were selected for qualitative comparison.

113

114 Model Performance 
Machine learning model performance was evaluated with different metrics:

116 accuracy, recall, precision, specificity, F1-score, area under receiver operating

117 characteristic curve, Cohen's kappa, and the Matthews correlation coefficient. The

118 statistics were calculated for both training data with five-fold cross validation, to evaluate

119 training performance, as well as in external testing set, to evaluate model performance

120 in predicting data outside the training set.

122 Principal Component Analysis

123 Principal Component Analysis (PCA) was computed for both the SARS-CoV-2

124 data set as well as SARS-CoV-2 with different compound libraries to assess its

125 chemical space. The scikit-learn ${ }^{36}(0.22 .2)$ PCA algorithm was used to reduce feature

126 dimensionality to three using different molecular descriptors (MW, MolLogP, NR, NArR,

127 NRB, HBA, HBD) and also with EFCP6 fingerprints. Molecular descriptors and

128 fingerprints were generated from the cheminformatics library RDkit (2020.03.1).

130 Applicability and Reliability Domain Assessment

131 In order to check if it is valid to apply the model for compounds being predicted

132 and how reliable the predictions are, an applicability and reliability domain assessment

133 was performed. First, the compound applicability within the model is assessed

134 comparing its similarity with the model's data using both molecular and fingerprint

135 descriptors. If the molecule satisfies both criteria it is considered within the applicability

136 domain and goes to the reliability domain assessment. 
The first criterion for the applicability assessment is determined based on 138 whether it fits within the range of the key molecular descriptors of the training set (MW, 139 MolLogP, NRB, TPSA, HBA, HBD). If at least four properties lie within the maximum 140 and minimum values of the model's data, the molecule is considered similar and goes to 141 the next criterion. The second criterion relies on structural fragment-based similarity 142 measured with Tanimoto coefficient using MACCS fingerprints. The similarity of the 143 MACCS fingerprints for the query compound and all training data is computed using the

144 Tanimoto score. Only $5 \%$ of the training set compounds that are most similar to the 145 query compound is used for evaluation (i.e. if the training set has 100 molecules only 5 146 molecules with more similarity to the query compound are used for the next evaluation).

147 If the Tanimoto score exceeds 0.5 against the $5 \%$ of the training set compounds, the 148 model is considered to have enough structural fragments overlap with the query 149 compound and thus the compound goes onto the reliability assessment.

150 The reliability domain assessment implements k-means clustering methods 151 based on ECFC6 fingerprints to classify the predictions from very high to low reliability.

152 The reliability class depends on four criteria: distance from the major central point of the 153 training data, distance from the closest cluster, closest cluster density and closest 154 cluster distance within the chemical space. Each criterion has different weights and 155 scores, with the second and third having higher priority. If the compound scores 1 in 156 each criterion it is classified as very highly reliable, if that is not the case only the two 157 higher priority criteria are considered for the next classes. The compound is classified 158 as highly reliable if scores a total of 2 , moderately reliable if it scores between -1 and 2 159 or low reliability if it scores less than or equal to -1 in the two higher priority criteria. The 
160 scores for each criterion as well as its definition are extensively described in the

161 Supplemental Methods.

163 In vitro testing

164 Compounds were tested in a 10-point serial dilution experiment to determine the 50\%

165 inhibitory concentration $\left(\mathrm{IC}_{50}\right)$ and $50 \%$ cytotoxicity concentration $\left(\mathrm{CC}_{50}\right) \cdot 1,000$ HeLa-

166 ACE2 cells/well were added into 384-well plates with compounds in a volume of $25 \mathrm{nl}$.

167 The final concentrations of compound ranged from $78 \mathrm{nM}-40 \mu \mathrm{M}$. $4 \mathrm{~h}$ post seeding 500

168 pfu SARS-CoV-2 (Washington strain USA-WA1/2020), BEl Resources NR-52281 were

169 added to each well at a $\mathrm{MOI}=0.5$. Twenty-four hours post infection cells were fixed with

$1704 \%$ formaldehyde solution. The cells were then treated with a Primary ab: human

171 polyclonal plasma (COVID-19 patient); Secondary ab: goat anti-human IgG coupled

172 with HRP. Images were acquired with ImageXpress MicroXL (bright field); Custom

173 Module developed in MetaXpress was used for automated count of total cells and

174 infected cells. Antiviral activity was assessed based on the infection ratio (number of 175 infected cells/total number of cells) in comparison with the average infection ration of 176 the untreated controls.

\section{Results}

179 Data Curation

180 In vitro SARS-CoV-2 data was initially collated from five drug repurposing studies

181 leading to a data set of 63 molecules with mean activity of $15.94 \pm 22.45 \mu \mathrm{M}^{8,9,12,14,15}$.

182 The external testing set collated from different studies has 30 molecules and a mean 
183 activity of $34 \pm 42 \mu \mathrm{M}^{11,21-25}$. Most assays were performed with different Vero cell lines,

184 inhibition was measured with viral RNA quantification, cytopathogenic effects or

185 immunofluorescence methods with $\mathrm{MOI}$ and incubation time varying from 0.01-0.05 and

$186 \quad 24-72$ hrs respectively (Figure S1). The threshold set for activity classification by the

187 Bayesian model generated with $\mathrm{AC}$ was $6.65 \mu \mathrm{M}$, with a final ratio of $52 \%$ actives in the

188 training set and $37 \%$ in the external test set. The molecules in both training and test set

189 are available in the supplemental data.

191 Machine Learning Models

Machine learning models were developed with $A C$ as well as several other

193 methods available to us. This five-fold cross validation comparison shows the different

194 prediction statistics for all machine learning algorithms implemented with the training

195 data only (Table 1). AC outperformed all of them at the threshold of $6.65 \mu \mathrm{M}$ with Rf

196 coming the closest. These models were chosen for further external testing predictions.

Table 1 - Five-fold cross validation statistics for all SARS-CoV-2 machine

199 learning models implemented using ECFP6 fingerprints.

\begin{tabular}{|c|c|c|c|c|c|c|c|c|}
\cline { 2 - 9 } \multicolumn{1}{c|}{} & ACC & AUC & CK & MCC & Pr & Recall & Sp & F1 \\
\hline AC & 0.81 & 0.78 & 0.62 & 0.64 & 0.78 & 0.88 & 0.73 & 0.83 \\
\hline rf & 0.75 & 0.74 & 0.49 & 0.5 & 0.73 & 0.82 & 0.67 & 0.77 \\
\hline knn & 0.71 & 0.71 & 0.43 & 0.42 & 0.71 & 0.76 & 0.67 & 0.74 \\
\hline svc & 0.7 & 0.69 & 0.39 & 0.4 & 0.68 & 0.79 & 0.6 & 0.73 \\
\hline bnb & 0.68 & 0.68 & 0.36 & 0.36 & 0.7 & 0.7 & 0.67 & 0.7 \\
\hline
\end{tabular}




\begin{tabular}{|c|c|c|c|c|c|c|c|c|} 
ada & 0.64 & 0.63 & 0.27 & 0.26 & 0.65 & 0.67 & 0.6 & 0.66 \\
\hline $\mathbf{D L}$ & 0.65 & 0.65 & 0.3 & 0.3 & 0.66 & 0.67 & 0.63 & 0.66 \\
\hline
\end{tabular}

ACC: Accuracy, AUC: Area under curve, CK: Cohen's Kappa, MCC: Matthews

Bayes, ada: AdaBoost Decision trees, rf: Random Forest, svc: support vector

\section{External Validation}

The performance of the machine learning models on the external testing data is shown in Table 2. The external validation was used to measure model performance in

209 data from different studies outside the training set. Svc and knn had slightly better

210 statistics compared to all other models, with the best balance between recall and

211 specificity.

Table 2 - Prediction statistics with the external data for all SARS-CoV-2

214 machine learning models implemented

\begin{tabular}{|c|c|c|c|c|c|c|c|c|}
\cline { 2 - 9 } \multicolumn{1}{c|}{} & ACC & AUC & CK & MCC & Pr & Recall & Sp & F1 \\
\hline AC & 0.62 & 0.58 & 0.17 & 0.17 & 0.50 & 0.40 & 0.76 & 0.44 \\
\hline rf & 0.63 & 0.57 & 0.10 & 0.11 & 0.42 & 0.30 & 0.80 & 0.35 \\
\hline knn & 0.67 & 0.6 & 0.21 & 0.21 & 0.50 & 0.40 & 0.80 & 0.44 \\
\hline svc & 0.70 & 0.57 & 0.34 & 0.34 & 0.54 & 0.60 & 0.75 & 0.57 \\
\hline bnb & 0.50 & 0.49 & -0.09 & -0.09 & 0.27 & 0.30 & 0.60 & 0.28 \\
\hline
\end{tabular}




\begin{tabular}{|c|c|c|c|c|c|c|c|c|} 
ada & 0.53 & 0.49 & 0.00 & 0.00 & 0.33 & 0.40 & 0.60 & 0.36 \\
\hline $\mathbf{D L}$ & 0.63 & 0.56 & 0.15 & 0.15 & 0.44 & 0.40 & 0.75 & 0.42 \\
\hline
\end{tabular}

\section{Chemical Space}

218 The PCA of the model training set alone shows that the SARS-CoV-2 chemical

219 space is well distributed with active and inactive molecules well mixed when analyzed

220 using either molecular and fingerprint descriptors. When compared with Prestwick

221 Chemical Library (PwCL), a library of predominantly FDA approved drugs, the SARS-

222 CoV-2 data lie within a big cluster with molecular descriptors and is more widely 223 distributed when using the fingerprint descriptors. 
Figure 1 - PCA of the SARS-CoV-2 set with Molecular Descriptors (A), and

ECFP6 (B). Red Spheres - Active, Grey Spheres - Inactive. PCA of SARS-CoV-2 set

227 and Prestwick Chemical Library (PwCL) with molecular descriptors (C), and ECFP6 (D).

228 Red Spheres - SARS-CoV-2, Grey Spheres - PwCL

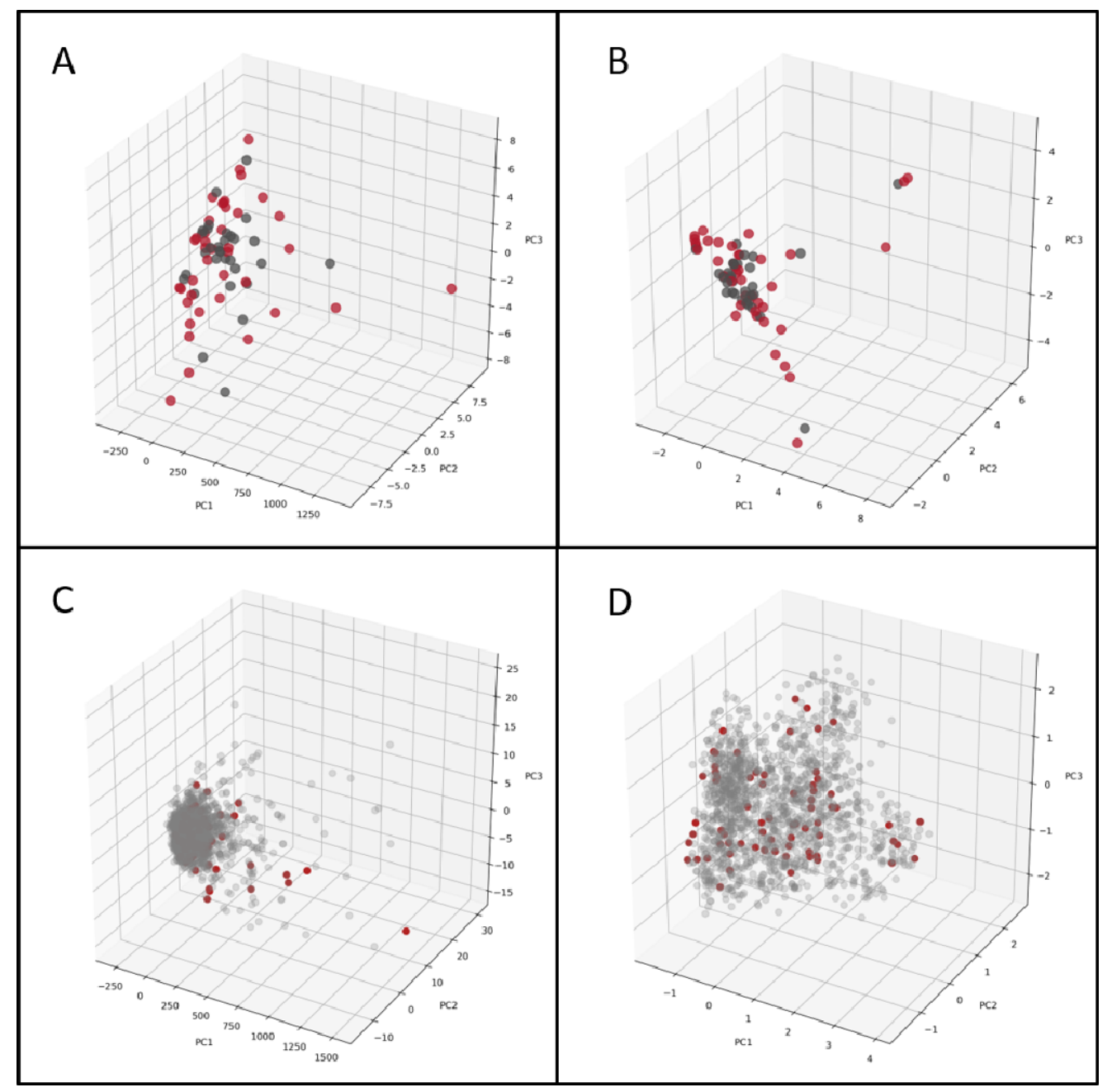

230 Applicability and Reliability Domain Assessment of External Test Set

The applicability and reliability domain assessment of the external test set was

232 determined for each molecule as described in the methods to see how the test set

233 compares with the training data. Molecules in the applicability domain are considered

234 suitable for the model predictions due to similarity based on structural and molecular 
235 properties with the training data, whereas the reliability value is a measurement of how

236 reliable the predictions are and uses different clustering metrics to determine its value.

237 From 30 molecules in the external test set, 22 were within the training data

238 applicability domain and had their reliability value calculated. Most molecules that fell

239 within the applicability domain had high or very high reliability values, with only $36 \%$

240 showing moderate reliability, so, most molecules obey the similarity criteria and are not

241 far away from dense clusters. In comparison, with the Assay Central applicability score,

242 which accounts only for structural similarity of the query compound with the training

243 data, only 10 molecules were considered within the domain with a higher reliability,

244 suggesting it is likely more conservative. Indeed, with the external test and training set

245 PCA we can see that most molecules superimpose with few of them distant from each

246 other (Figure S1). Therefore, similarity together with clustering methods are more

247 suitable for applicability and reliability assessment compared with only structural

248 similarity, as seen by the PCA.

250 Prospective Prediction

251 A selection of FDA approved drugs available to us in our relatively small in-house

252 compound collection of hundreds of molecules was scored with the AC Bayesian model.

253 A selection of some of the best scoring molecules (Table 3) was used to identify and

254 prioritize compounds for in vitro testing. AC Applicability score is the similarity of the

255 compound with the training data, compounds are ranked by reliability which may

256 provide some degree of confidence in these predictions. 


\begin{tabular}{cccc}
\hline Name & $\begin{array}{c}\text { Prediction } \\
\text { Score }\end{array}$ & $\begin{array}{c}\text { AC Applicability } \\
\text { Score }\end{array}$ & Reliability \\
\hline CPI1062 & 0.67 & 0.5 & High \\
\hline CPI1066 & 0.62 & 0.38 & High \\
\hline CPI1004 & 0.62 & 0.39 & Moderate \\
\hline CPI1012 & 0.70 & 0.70 & Moderate \\
\hline CPI1155 & 0.70 & 0.40 & Moderate \\
\hline CPI1175 & 0.65 & 0.41 & Low \\
\hline CPI1153 & 0.7 & 0.7 & 0.40
\end{tabular}

258 Table 3 - Prospective prediction compounds predicted and prioritized for testing.

260 In vitro Inhibition Assays of Predicted Compounds

261 Antiviral activity testing in the HeLa-ACE2 cells demonstrated that CPI1155 and

262 CPI1062 have antiviral activity with $\mathrm{IC}_{50}$ values of $8.4 \mu \mathrm{M}$ and $540 \mathrm{nM}$ (Figure 2),

263 respectively. The cell viability of these compounds was also tested, with both $\mathrm{CC}_{50}$

264 higher than $40 \mu \mathrm{M}$. Other compounds did not inhibit viral replication in HeLa cells or had

265 appreciable cytotoxicity.

$267 \quad$ Figure 2 - Preliminary dose response curves for a) CPI1155 and b) CPI1062. 
a)

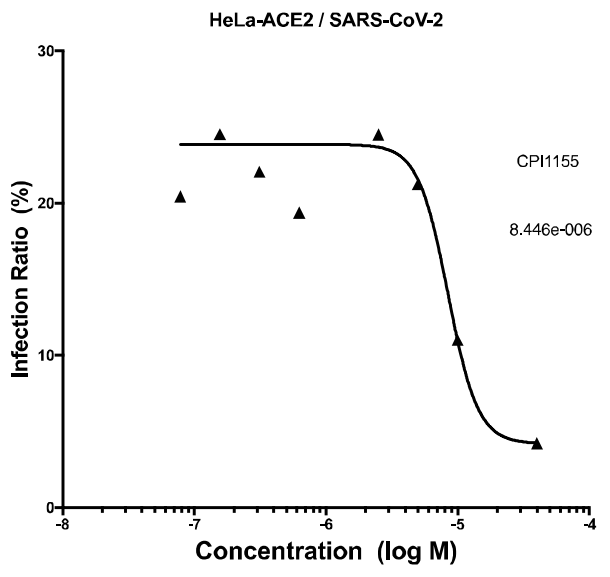

b)

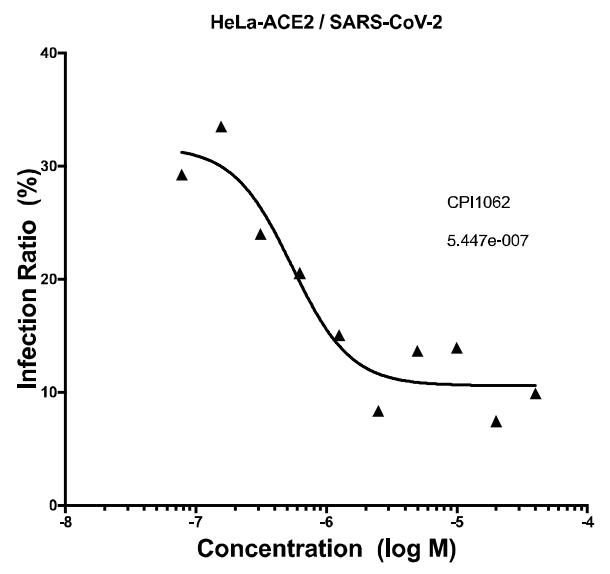

271 Discussion

272 One of the challenges for addressing novel viral outbreaks is selection of drugs

273 to test. Testing capacity, even for in vitro antiviral activities is likely to be low at the 274 onset of an outbreak, making compound selection even more critical in this situation. In

275 the case of SARS-CoV-2, the initial focus was on molecules that had previously shown 276 activity against SARS or MERS ${ }^{37,38}$. The training set for the current model is therefore

277 not a random sampling of drug property space. When compared with the PwCL, a

278 library of mostly FDA approved drugs, all molecules superimpose in the property space

279 highlighting the model suitability for drug repurposing. Even with a relatively small

280 training dataset the machine learning models evaluated have shown acceptable five-

281 fold cross validation statistics, with almost all metrics greater than random and ROC

$282>0.75$ for AC (Table 1). When compared with different machine learning methods AC

283 outperforms all of them in the SARS-CoV-2 training set, but this may be due to the

284 threshold for all models being set as optimal for AC. However, choosing different values

285 could imbalance the training set and remove important compounds from the active 286 group. 
More important than a good performance in the training set is the performance on external data, since most prospective predictions will occur for molecules outside training data. For external validation all models had intermediate performance, with

290 ROC of 0.6. Taking into account the small number of molecules and that some test set

291 molecules lie outside the applicability domain, the performance is acceptable. Different

292 from the training set performance, svc had the highest overall score, predicting $60 \%$ of

293 the active molecules despite its modest statistics in five-fold cross validation. The good

294 performance of svc in predicting biological activity is in accordance with several studies

295 that show good performance in different datasets $28,32,35,39$. Therefore, the models

296 described here are suitable for initial prospective predictions.

297 The applicability and reliability assessment shows that $73 \%$ of the test set 298 molecules lie within the model applicability domain with high to moderate reliability, so

299 poor performance in external validation occurs because there isn't a clear boundary in 300 the model's feature space that can correctly classify external data. Increasing the 301 number of molecules might include new features in both actives and inactive molecules 302 which can increase model performance in both training and external data.

303 The training and test set described herein can be merged to increase data set 304 size and applicability domain. The AC model with merged training and test data has 305 slightly worse statistics (ACC: 0.76, AUC:0.79, CK: 0.53, MCC: 0.75, Pr: 0.76, Recall: 306 0.76, Sp: 0.77, F1: 0.76), but a higher applicability domain. The PCA confirms this wide 307 chemical property space (Figure S1), the PCA of this updated model is much more 308 balanced and broader than the previous one (Figure S2) versus Figure 1B. Without 309 some form of external validation, we cannot assess how predictions of compounds 
310 outside the applicability domain perform, as model statistics were comparable it is

311 expected that compounds outside this would obviously have unreliable predictions,

312 however this may be offset by a higher domain which can increase reliability of some

313 compounds.

314 The molecules of the dataset do not have a common scaffold, but there are

315 several common structural features that occur in active/inactive molecules that can be

316 highlighted, such as tertiary amines and aliphatic chains in active molecules and phenyl

317 rings and peptide molecule features in inactive molecules (Figure S3). These most

318 common active features appear in chloroquine, tripanarol and tilorone, while the inactive

319 features appear in darunavir, amprenavir and ritonavir (Figure 3). The lack of common

320 scaffolds and features that appears in more than $30 \%$ of the active or inactive

321 molecules shows how different and diverse the active molecules are, which turn

322 classification models for these molecules into a relatively difficult task.

324 Figure 3- Common Active/Inactive structure features of the SARS-CoV-2 dataset

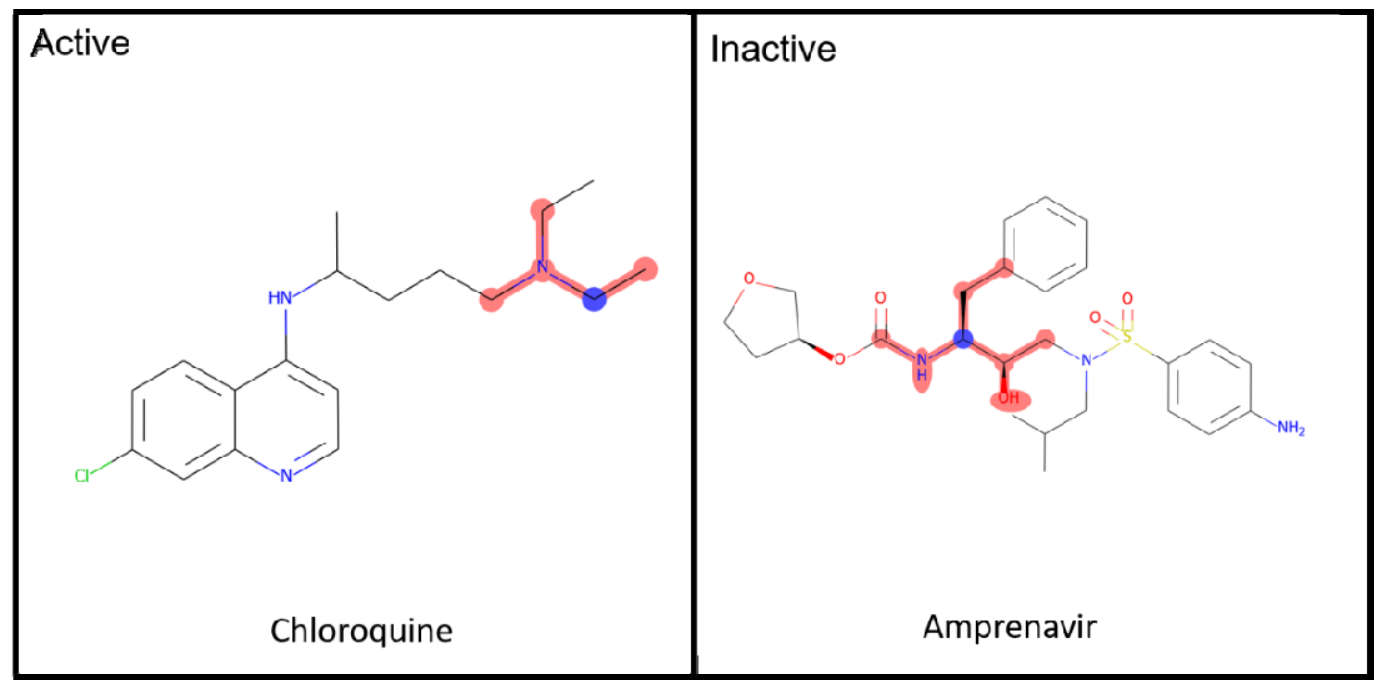


The performance of a predictive model is highly dependent on the curation and

328 data used. One of the main problems that comes from building models with biological

329 data from different laboratories is data reproducibility and assay standardization ${ }^{40}$. Cell

330 based assays of viral infections have many parameters that can affect the compound

331 potency, e.g., cell lines, $\mathrm{MOI}$, assay readout ${ }^{41}$. From all inhibition assays for SARS-CoV-

3322 collated to date, most studies use MOI of $0.01-0.05$ (73\% of data), different Vero cell

333 lines (77\% of data) and qRT-PCR ( $60 \%$ of data), however there is no clear definition of

334 compound addition time post infection (Figure S1).

335 Besides this, even assays with the same or similar conditions have differences in

336 'control' compounds such as chloroquine or remdesivir, showing a lack of data

337 reproducibility between laboratories, which can impact model building. If we keep only

338 studies with the most in common there is not enough data to build a model, while

339 merging all studies will have problems of different assay parameters. It was shown that

340 for Ebola infections in VeroE6 cells the change in the compound potency at different

341 time post infections are lower when using $\mathrm{MOI}$ of $0.01-0.1$ therefore, merging different

342 assays with the same cell line and low $\mathrm{MOI}$ is a good choice to avoid data 343 inconsistency ${ }^{41}$.

344 It should be noted that most of the in vitro data collated to date uses Vero or Vero

345 E6 cells for inhibition assays. Although these cells lines have high ACE2 expression

346 levels, they lack a TMPRSS2 gene. Priming of viral S proteins can occur with the host

347 cell protease TMPRSS2 and Cathepsin L and is essential for SARS-CoV-2 entry ${ }^{42,43}$.

348 Therefore, inhibition assays with cells that do not express TMPRSS2 should be avoided

349 as they might miss compounds that could inhibit the protein and instead find 
350 compounds that prevent virus entry by inhibiting only Cathepsin L. In order to avoid

351 these problems with the TMPRSS2 and Cathepsin $\mathrm{L}$ gene, cell lines like Calu-3 or

352 modified Vero cell lines should be used instead. ${ }^{44}$

$353 \quad$ From the 7 compounds prioritized for testing in our laboratory using the machine

354 learning model, CPI1155 and CPI1062 showed antiviral activity against SARS-CoV-2

355 infections in HeLa-ACE2 cells. Like Vero cells, HeLa does not express TMPRSS2,

356 therefore compounds might need to be be retested in different cell lines to see whether

357 or not the expression of TMPRSS2 affects compound activity. ${ }^{45}$

358 As new data is continually being published the machine learning models can be

359 updated to increase performance in terms of both training and external test set

360 validation. The very latest model for SARS-CoV-2 is available at www.assaycentral.org.

361 In the meantime, we have shown these models perform well with internal cross

362 validation, external validation as well as prospective prediction, enabling us to find

363 additional active molecules. These models should be used to prioritize compounds

364 which have both a high prediction score and reliability as described herein. This will be

365 expected to return more reliable predictions that together with drug discovery expertise

366 can help prioritize compounds in future for in vitro testing.

368 Acknowledgements

369 We would like to kindly acknowledge Dr. Nancy Baker and Ms. Natasha Baker for their

370 help in collating recently SARS-CoV-2 published data. We also thank Biovia for

371 supplying Discovery Studio. Per Subcontract: "This material is based upon work

372 supported by the Defense Advanced Research Projects Agency (DARPA) under 
373 Contract No. HR001119C0108." Per DISTAR Form: "The views, opinions, and/or

374 findings expressed are those of the author(s) and should not be interpreted as

375 representing the official views or policies of the Department of Defense or the U.S.

376 Government."

377

378 Funding

379 We kindly acknowledge NIH funding: R44GM122196-02A1 from NIGMS (PI - Sean

380 Ekins) and support from DARPA (HR0011-19-C-0108; PI: P. Madrid) is gratefully

381 acknowledged. Distribution Statement "A" (Approved for Public Release, Distribution

382 Unlimited). The views, opinions, and/or findings expressed are those of the author and

383 should not be interpreted as representing the official views or policies of the Department

384 of Defense or the U.S. Government. FAPESP funding: 2019/25407-2 (PI - Glaucius

385 Oliva).

387 Conflicts of interest

388 SE is CEO and owner of Collaborations Pharmaceuticals, Inc. DHF, KMZ, TRL, AP are 389 employees of Collaborations Pharmaceuticals, Inc.

\section{References}

392 1. Wu F, Zhao S, Yu B, et al. A new coronavirus associated with human respiratory

393 disease in China. Nature. 2020;579(7798):265-269. doi:10.1038/s41586-020-

$394 \quad 2008-3$

395 2. Gorbalenya AE, Baker SC, Baric RS, et al. The species Severe acute respiratory 396 syndrome-related coronavirus: classifying 2019-nCoV and naming it SARS-CoV-

397 2. Nat Microbiol. 2020;5(March). doi:10.1038/s41564-020-0695-z 
398 3. WHO. Naming the coronavirus disease (COVID-2019) and the virus that causes it.

4004 4ractice BB. Coronavirus disease 2019. World Heal Organ. 2020;2019(April):2633. doi:10.1001/jama.2020.2633

5. Kupferschmidt K, Cohen J. Race to find COVID-19 treatments accelerates. Science (80- ). 2020;(March). doi:10.1126/science.367.6485.1412

6. Harrison C. Coronavirus puts drug repurposing on the fast track. Nat Biotechnol. February 2020. doi:10.1038/d41587-020-00003-1

7. Baker NC, Ekins S, Williams AJ, Tropsha A. A bibliometric review of drug repurposing. Drug Discov Today. 2018;23(3):661-672. doi:10.1016/j.drudis.2018.01.018

8. Jeon S, Ko M, Lee J, et al. Identification of antiviral drug candidates against SARS-CoV-2 from FDA-approved drugs. bioRxiv. 2020:2020.03.20.999730. doi:10.1101/2020.03.20.999730

9. Weston S, Haupt R, Logue J, Matthews K, Frieman MB. FDA approved drugs with broad anti-coronaviral activity inhibit SARS-CoV-2 in vitro. 2020;(3).

10. Sheahan TP, Sims AC, Zhou S, et al. An orally bioavailable broad-spectrum antiviral inhibits SARS-CoV-2 and multiple endemic, epidemic and bat coronavirus. bioRxiv. 2020;(153):2020.03.19.997890.

12. Jin Z, Du X, Xu Y, et al. Structure of Mpro from COVID-19 virus and discovery of its inhibitors. bioRxiv. 2020:2020.02.26.964882. doi:10.1101/2020.02.26.964882

13. Zhang J, Ma X, Yu F, et al. Teicoplanin potently blocks the cell entry of 2019nCoV. bioRxiv. 2020.

425 14. Wang M, Cao R, Zhang L, et al. Remdesivir and chloroquine effectively inhibit the recently emerged novel coronavirus (2019-nCoV) in vitro. Cell Res.

428 15. Liu J, Cao R, Xu M, et al. Hydroxychloroquine, a less toxic derivative of 
chloroquine, is effective in inhibiting SARS-CoV-2 infection in vitro. Cell Discov. 2020;6(1):16. doi:10.1038/s41421-020-0156-0

16. Soares VC, Gomes S, Temerozo JR, et al. Atazanavir inhibits SARS-CoV-2 replication and pro-inflammatory cytokine production. 2020.

17. Cherkasov A, Muratov EN, Fourches D, et al. QSAR modeling: Where have you been? Where are you going to? J Med Chem. 2014;57(12):4977-5010. doi:10.1021/jm4004285

18. Lima AN, Philot EA, Trossini GHG, Scott LPB, Maltarollo VG, Honorio KM. Use of machine learning approaches for novel drug discovery. Expert Opin Drug Discov. 2016;11(3):225-239. doi:10.1517/17460441.2016.1146250

19. Ekins S, Puhl AC, Zorn KM, et al. Exploiting machine learning for end-to-end drug discovery and development. Nat Mater. 2019;18(5):435-441. doi:10.1038/s41563019-0338-z

20. A P. Vanquishing the Virus: 160+ COVID-19 Drug and Vaccine Candidates in Development. https://www.genengnews.com/a-lists/vanquishing-the-virus-160covid-19-drug-and-vaccine-candidates-in-development/. Published 2020. Accessed May 3, 2020.

446 21. Riva L, Yuan S, Yin X, et al. A Large-scale Drug Repositioning Survey for SARSCoV-2 Antivirals. 2020.

22. Su H, Yao S, Zhao W, Li M, Liu J, Shang W. Discovery of baicalin and baicalein as novel, natural product inhibitors of SARS-CoV-2 3CL protease in vitro. 2020:129.

24. Touret F, Gilles M, Barral K, Nougairède A, Decroly E. In vitro screening of a FDA approved chemical library reveals potential inhibitors of SARS-CoV-2 replication. bioRxiv. 2020.

458 25. Xu T. Indomethacin has a potent antiviral activity against SARS CoV-2 in vitro and canine coronavirus in vivo Abstract $\square: 2020$;(December 2019). 
26. Ekins S, Gerlach J, Zorn KM, Antonio BM, Lin Z, Gerlach A. Repurposing Approved Drugs as Inhibitors of $\mathrm{K}(\mathrm{v}) 7.1$ and $\mathrm{Na}(\mathrm{v}) 1.8$ to Treat Pitt Hopkins Syndrome. Pharm Res. 2019;36(9):137. doi:10.1007/s11095-019-2671-y

27. Dalecki AG, Zorn KM, Clark AM, et al. High-throughput screening and Bayesian machine learning for copper-dependent inhibitors of Staphylococcus aureus. Metallomics. 2019;11(3):696-706. doi:10.1039/c8mt00342d

28. Zorn KM, Lane TR, Russo DP, Clark AM, Makarov V, Ekins S. Multiple Machine Learning Comparisons of HIV Cell-based and Reverse Transcriptase Data Sets. Mol Pharm. 2019;16(4):1620-1632. doi:10.1021/acs.molpharmaceut.8b01297

29. Anantpadma M, Lane T, Zorn KM, et al. Ebola Virus Bayesian Machine Learning Models Enable New in Vitro Leads. ACS omega. 2019;4(1):2353-2361. doi:10.1021/acsomega.8b02948

30. Wang P-F, Neiner A, Lane TR, Zorn KM, Ekins S, Kharasch ED. Halogen Substitution Influences Ketamine Metabolism by Cytochrome P450 2B6: In Vitro and Computational Approaches. Mol Pharm. 2019;16(2):898-906. doi:10.1021/acs.molpharmaceut.8b01214

31. Hernandez HW, Soeung M, Zorn KM, et al. High Throughput and Computational Repurposing for Neglected Diseases. Pharm Res. 2018;36(2):27. doi:10.1007/s11095-018-2558-3

32. Russo DP, Zorn KM, Clark AM, Zhu H, Ekins S. Comparing Multiple Machine Learning Algorithms and Metrics for Estrogen Receptor Binding Prediction. Mol Pharm. 2018;15(10):4361-4370. doi:10.1021/acs.molpharmaceut.8b00546

33. Lane T, Russo DP, Zorn KM, et al. Comparing and Validating Machine Learning Models for Mycobacterium tuberculosis Drug Discovery. Mol Pharm. 2018;15(10):4346-4360. doi:10.1021/acs.molpharmaceut.8b00083

34. Sandoval PJ, Zorn KM, Clark AM, Ekins S, Wright SH. Assessment of SubstrateDependent Ligand Interactions at the Organic Cation Transporter OCT2 Using Six Model Substrates. Mol Pharmacol. 2018;94(3):1057-1068. doi:10.1124/mol.117.111443

35. Lane T, Russo DP, Zorn KM, et al. Comparing and Validating Machine Learning Models for Mycobacterium tuberculosis Drug Discovery. Mol Pharm. 
2018;15(10):4346-4360. doi:10.1021/acs.molpharmaceut.8b00083

36. Varoquaux G, Buitinck L, Louppe G, Grisel O, Pedregosa F, Mueller A. Scikitlearn. GetMobile Mob Comput Commun. 2015;19(1):29-33. doi:10.1145/2786984.2786995

37. Weston S, Haupt R, Logue J, Matthews K, Frieman M. FDA approved drugs with broad anti-coronaviral activity inhibit SARS-CoV-2 in vitro. bioRxiv. 2020;(3):2020.03.25.008482. doi:10.1101/2020.03.25.008482

38. Coleman CM, Frieman MB. Coronaviruses: Important Emerging Human Pathogens. J Virol. 2014;88(10):5209-5212. doi:10.1128/jvi.03488-13

39. Korotcov A, Tkachenko V, Russo DP, Ekins S. Comparison of Deep Learning with Multiple Machine Learning Methods and Metrics Using Diverse Drug Discovery Data Sets. Mol Pharm. 2017;14(12):4462-4475. doi:10.1021/acs.molpharmaceut.7b00578

40. Fourches D, Muratov E, Tropsha A. Trust, but Verify II: A Practical Guide to Chemogenomics Data Curation. J Chem Inf Model. 2016;56(7):1243-1252. doi:10.1021/acs.jcim.6b00129

41. Postnikova E, Cong Y, DeWald LE, et al. Testing therapeutics in cell-based assays: Factors that influence the apparent potency of drugs. PLoS One. 2018;13(3):1-18. doi:10.1371/journal.pone.0194880

43. Ou X, Liu Y, Lei X, et al. Characterization of spike glycoprotein of SARS-CoV-2 on virus entry and its immune cross-reactivity with SARS-CoV. Nat Commun. 2020;11(1):1620. doi:10.1038/s41467-020-15562-9

44. Matsuyama S, Nao N, Shirato K, et al. Enhanced isolation of SARS-CoV-2 by

45. Shirato K, Kanou K, Kawase M, Matsuyama S. Clinical Isolates of Human TMPRSS2-expressing cells. Proc Natl Acad Sci. 2020:202002589. 
bioRxiv preprint doi: https://doi.org/10.1101/2020.06.16.154765; this version posted June 16, 2020. The copyright holder for this preprint (which was not certified by peer review) is the author/funder, who has granted bioRxiv a license to display the preprint in perpetuity. It is made available under aCC-BY-NC-ND 4.0 International license.

523

524 